\title{
M 82: starburst Rosetta Stone
}

\author{
Natascha M. Förster Schreiber \\ Sterrewacht Leiden, \\ Postbus 9513, NL-2300 RA Leiden, Nederland \\ Reinhard Genzel, Dieter Lutz \\ Max-Planck-Institut für extraterrestrische Physik, \\ Postfach 1312, D-85741 Garching-bei-München, BRD \\ Amiel Sternberg \\ School of Physics and Astronomy, Tel Aviv University, \\ Ramat Aviv, Tel Aviv 69978, Israel
}

\begin{abstract}
We present evolutionary synthesis modeling of the nuclear regions of the starburst galaxy M 82, based on near-infrared integral field spectroscopy and mid-infrared ISO spectroscopy. Our data indicate the occurrence of two distinct starburst episodes in the central $500 \mathrm{pc}$ about 8-15 Myr and $5 \mathrm{Myr}$ ago, each lasting a few million years only. The first burst was most intense within $50 \mathrm{pc}$ of the nucleus while the second burst took place in a circumnuclear ring of radius $\sim 85 \mathrm{pc}$ and along the stellar bar at larger radii. These recent episodes succeeded earlier starburst activity $\sim 1 \mathrm{kpc}$ away from the nucleus and peaking $650 \mathrm{Myr}$ ago, as traced by the luminous star clusters studied by de Grijs et al. (2001). The combined results of both studies reveal complex evolution of starburst activity in M 82 which is consistent with a tidally-induced bar-driven scenario.
\end{abstract}

\section{Introduction}

In recent years, it has become evident that starburst galaxies are important constituents of the Universe at all accessible redshifts. However, a detailed and quantitative understanding of the starburst phenomenon is still lacking. Crucial issues still open include the initial mass function (IMF) of the stars formed in starburst environments, the evolution of starburst activity, and its triggering and quenching mechanisms. Progress has been hindered by the scarcity of spatially detailed observations and by large dust obscuration often hampering high angular resolution studies at optical/UV wavelengths. Investigations of local starburst galaxies thus remain extremely relevant.

$\mathrm{M} 82$, as one of the nearest $\left(3.3 \mathrm{Mpc}\right.$, implying $1^{\prime \prime} \approx 16 \mathrm{pc}$; Freedman \& Madore 1988) and most studied starburst, undoubtedly represents a Rosetta Stone in the quest to understand the starburst phenomenon. Extensive studies 
of M 82 have been carried out at all accessible wavelengths, providing a wealth of observational data for a detailed and consistent characterization of its starburst activity. The central $500 \mathrm{pc}$ of M 82 harbour the most active regions; this 'starburst core' is severely obscured by large amounts of dust partly due to a high inclination of $\sim 80^{\circ}$. A prominent nucleus, a stellar disk, and a kiloparsec-long stellar bar are revealed in near-infrared continuum light. Circumnuclear concentrations of molecular gas surround the starburst core at a radius of $\sim 400 \mathrm{pc}$ while the HII regions are distributed mainly in a ring of radius $85 \mathrm{pc}$ and along the stellar bar at larger radii. An important series of young compact radio supernova remnants extends fairly uniformly along the galactic plane over $600 \mathrm{pc}$ and a bipolar outflow traces a starburst wind out to several kiloparsecs. Over 200 compact and luminous stellar clusters have been resolved by optical HST imaging across the central $\sim 1 \mathrm{kpc}$. Beginning with the seminal paper by Rieke et al. (1980), several authors have used evolutionary synthesis to constrain the star formation parameters and history in M 82. However, most models were applied to the starburst core as a whole (e.g., Rieke et al. 1993) or to selected stellar clusters (Satyapal et al. 1997), and thus may only provide an incomplete picture. More exhaustive reviews are given by, e.g., Telesco (1988), Rieke et al. (1993), and de Grijs, O'Connell \& Gallagher (2001).

We summarize here our results of near-infrared integral field spectroscopy and mid-infrared spectroscopy of the most active starburst regions of M 82, focussing on the star formation history as traced by massive stars. The nearand mid-infrared regimes are rich in starburst signatures and probe deep into obscured star-forming regions (e.g., $A_{2 \mu m} \simeq 1 / 10 A_{V}$ ). Our spatially and spectrally detailed data allowed us to model the observed regions 'continuously' in space on scales of $\sim 25 \mathrm{pc}$ and in time up to $\sim 50 \mathrm{Myr}$ ago. We interpret our results together with those of de Grijs et al. (2001) on the 'fossil' starburst regions further out in the disk. The combination of both studies provides unique quantitative constraints for the evolution of starburst activity in M 82. A full account of our work is given by Förster Schreiber et al. (2001; 2002).

\section{Probing M 82's obscured starburst: near- and mid-infrared data}

The near-infrared data were obtained with the 3D integral field spectrometer (Weitzel et al. 1996) of the Max-Planck-Institut für extraterrestrische Physik (MPE). We mapped in $H$ - and $K$-band a region of $\sim 16^{\prime \prime} \times 10^{\prime \prime}(260 \times 160 \mathrm{pc})$ including the nucleus and extending to the west along the galactic plane out to the inner edge of the molecular ring. The reduced data have an effective angular resolution of $1.5^{\prime \prime}(25 \mathrm{pc})$ and spectral resolution of $R \equiv \lambda / \Delta \lambda \simeq 1000$. We complemented these observations with 2.4 - $45 \mu \mathrm{m}$ spectroscopy at $R \approx 500$ 1000 from the short wavelength spectrometer (sws, de Graauw et al. 1996) on board the Infrared Satellite Observatory ${ }^{1}$ (ISO, Kessler et al. 1996). The SwS $14^{\prime \prime} \times 20^{\prime \prime}$ to $20^{\prime \prime} \times 33^{\prime \prime}$ apertures are approximately centered on and include the area mapped with 3D.

\footnotetext{
${ }^{1}$ ISO is an ESA project with instruments funded by ESA Member States (especially the PI countries: France, Germany, the Netherlands, and the United Kingdom) and with the participation of ISAS and NASA. The SWS is a joint project of SRON and MPE.
} 

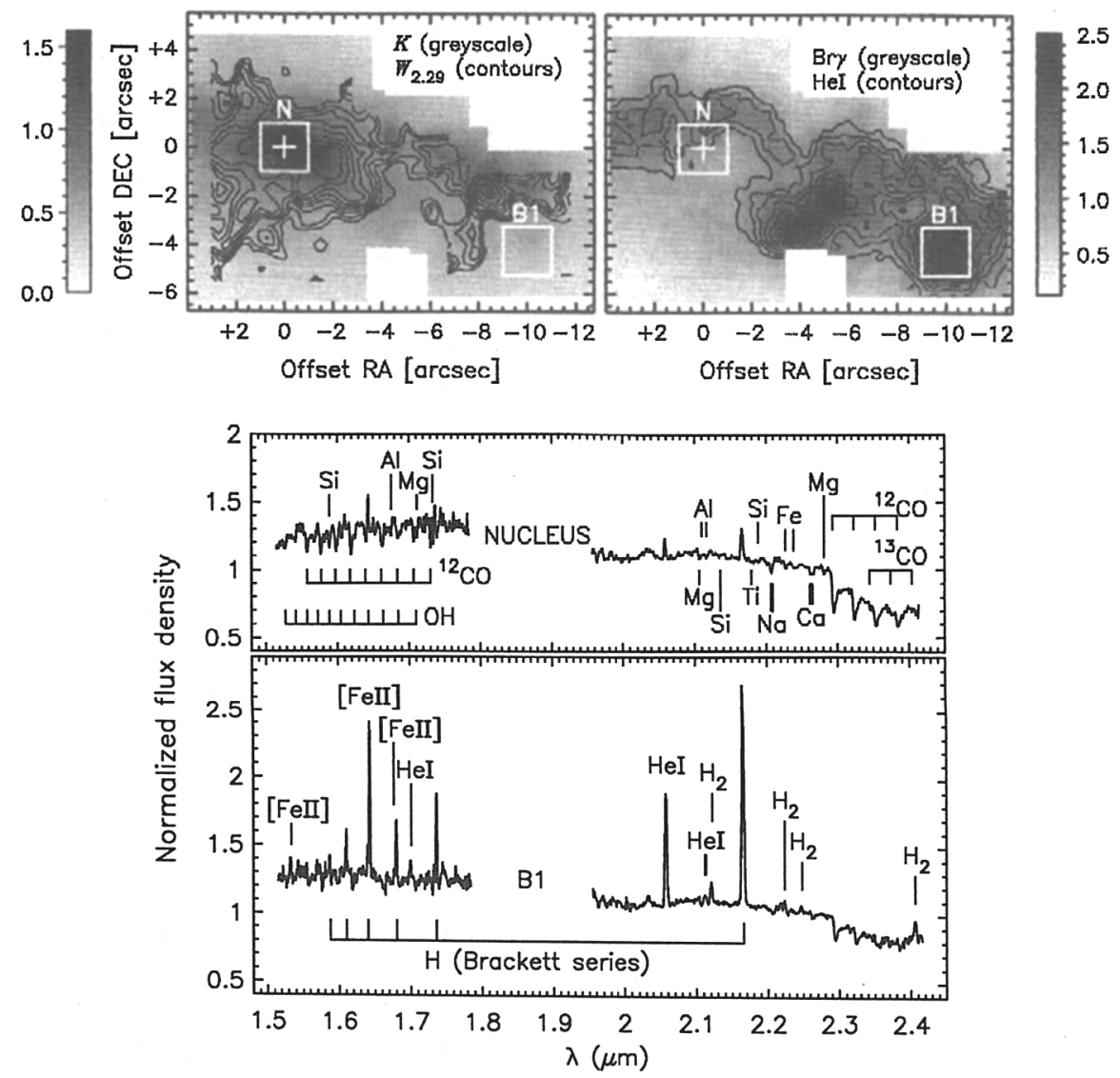

Figure 1. 3D maps of M 82 and spectra of the nucleus (cross, ' $N$ ') and region 'B1'. Greyscale levels are in $10^{-14} \mathrm{~W} \mathrm{~m}^{-2} \mu \mathrm{m}^{-1}$ arcsec ${ }^{-2}$ for the $K$-band map and $10^{-17} \mathrm{~W} \mathrm{~m}^{-2} \operatorname{arcsec}^{-2}$ for the $\operatorname{Br} \gamma$ map. Contours for the $W_{2.29}$ map range from $12 \AA$ to $16 \AA$ in steps of $0.5 \AA$, those for the He $\mathrm{I} 2.058 \mu \mathrm{m}$ linemap range from 0.5 to 1.5 in steps of 0.1 and units of $10^{-17} \mathrm{~W} \mathrm{~m}^{-2} \operatorname{arcsec}^{-2}$. The spectra are normalized to unity in the range $2.2875-2.2910 \mu \mathrm{m}$ (normalizing factors in $10^{-14} \mathrm{~W} \mathrm{~m}^{-2} \mu \mathrm{m}^{-1}$ are 6.52 for the nucleus and 1.78 for $\mathrm{B} 1$ ).

Among the key diagnostics provided by the 3D and sws data, we used $\mathrm{H}$ and $\mathrm{He}$ recombination lines and mid-infrared fine-structure lines of $\mathrm{Ne}, \mathrm{Ar}$, and $\mathrm{S}$ originating in $\mathrm{H}$ II regions to constrain the properties of the most massive stars present. We also used the equivalent widths of near-infrared stellar absorption features including the CO $2.29 \mu \mathrm{m}$ and $1.62 \mu \mathrm{m}$ bandheads $\left(W_{2.29}, W_{1.62}\right)$ to characterize the cool evolved star populations. Figure 1 presents selected 3D maps together with the $H$ - and $K$-band spectra of the central $35 \mathrm{pc}$ at the nucleus and of the brightest $\operatorname{Br} \gamma$ source $\sim 10^{\prime \prime}$ to the southwest ('B1').

The maps show distinct burst sites on scales as small as $25 \mathrm{pc}$ and important spatial variations in the strength of the absorption features and emission lines relative to the continuum. The distribution of $\mathrm{CO}$ bandhead equivalent widths follows well the $H$-and $K$-band emission. Comparison of the $W_{2.29}$ 

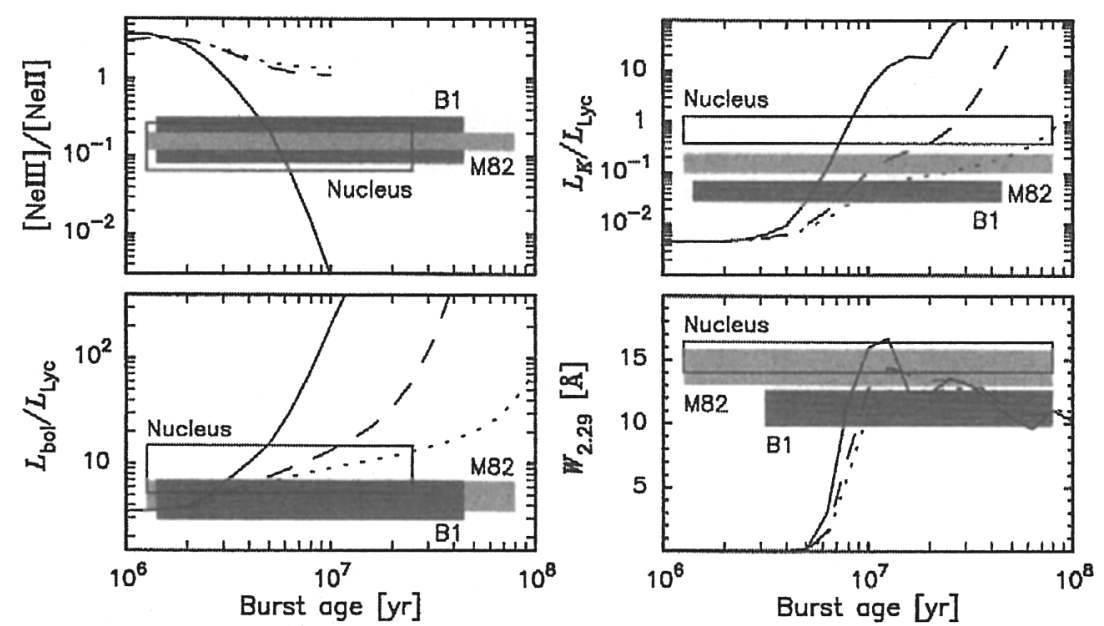

Figure 2. Comparison of the properties of selected regions in M82 with model predictions for a Salpeter IMF between 1 and $100 \mathrm{M}_{\odot}$ and burst decay timescales of 1,5 , and $20 \mathrm{Myr}$ (solid, dashed, and dotted curves). Measurements for the starburst core ('M 82'), the nucleus, and the Br $\gamma$ source B1 are indicated by horizontal bars of width corresponding to the uncertainties.

and $W_{1.62}$ with empirical stellar data (e.g., Origlia, Moorwood \& Oliva 1993; Förster Schreiber 2000) together with the mass-to- $K$-band light ratio $\left(M / L_{K}\right)$ indicate that red supergiants of temperatures $3600-4500 \mathrm{~K}$ dominate the nearinfrared continuum, with the cooler stars located at the nucleus and along a ridge extending up to just north of $\mathrm{B} 1$. The distribution of the ionized gas line emission contrasts sharply with that of the red supergiants light, with very little emission at the nucleus, prominent sources to the west, and little variation in the ratios of $\mathrm{He}$ to $\mathrm{H}$ recombination lines. From photoionization modeling using CLOUDY (Ferland 1996) and the non-LTE unified stellar atmospheres of Pauldrach, Hoffmann \& Lennon (2001), the nebular line ratios (e.g., $\mathrm{He}$ I $2.058 \mu \mathrm{m} / \mathrm{Br} \gamma$, [Ne III $15.6 \mu \mathrm{m} /[\mathrm{Ne}$ II] $12.8 \mu \mathrm{m}$ ) imply temperatures of $\sim 37100 \mathrm{~K}$ for the OB stars with small dispersion of $650 \mathrm{~K}$, suggesting that stars of masses $\gtrsim 30 \mathrm{M}_{\odot}$ have disappeared due to rapid aging of the starburst.

\section{Recent history of M 82: modeling the starburst core}

In order to constrain quantitatively the star formation history in the regions observed, we applied starburst models which combine evolutionary synthesis and photoionization modeling with STARS and CLOUDY (Sternberg 1998; Ferland 1996), and employ the Geneva tracks (e.g., Schaller et al. 1992) and the Pauldrach et al. (2001) atmospheres. Figure 2 compares the observational constraints for the nucleus, region $\mathrm{B} 1$, and the starburst core with model predictions. The Lyman continuum and bolometric luminosities $\left(L_{\mathrm{Lyc}}\right.$ and $\left.L_{\mathrm{bol}}\right)$ were derived from our $\mathrm{H}$ line fluxes and from the $12 \mu \mathrm{m}$ data of Telesco \& Gezari (1992), respectively. The models were computed for a Salpeter IMF $\mathrm{d} N / \mathrm{d} m \propto m^{-2.35}$ 


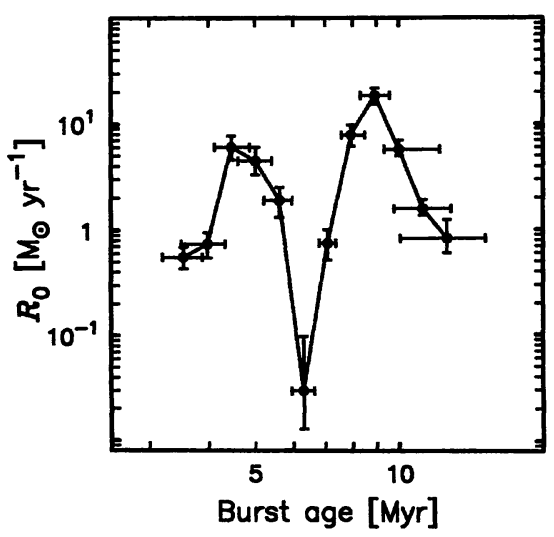

Figure 3. Star formation history in the central regions of M 82 reconstructed from the spatially-detailed modeling by integrating the burst intensity per age interval.

between 1 and $100 \mathrm{M}_{\odot}$ and various timescales $t_{\mathrm{sc}}$ for a star formation rate $R(t)=R_{0} \exp \left(-t / t_{\mathrm{sc}}\right)$.

Focussing on the diagnostics tracing the OB stars, the Ne line and $L_{\mathrm{bol}} / L_{\mathrm{Lyc}}$ ratios can only be reconciled with very short burst timescales of a few $10^{6} \mathrm{yr}$ at most and imply ages of 4-5 Myr. On the other hand, the strength of the $2.29 \mu \mathrm{m}$ CO bandhead produced exclusively in the atmosphere of cool stars implies systematically older ages $\gtrsim 8 \mathrm{Myr}$ and, for the nucleus, suggests $t_{\mathrm{sc}}<5 \mathrm{Myr}$. We adopted the younger age solutions from $W_{2.29}$; the very low $M / L_{K}$ ratio in the starburst core supports the presence of very young red supergiants throughout. The $L_{K} / L_{\text {Lyc }}$ ratios probing both hot main sequence and cool evolved stars indicate intermediate ages.

The various constraints are difficult to reproduce simultaneously with single bursts of any timescale. Models consisting of two successive short bursts are more successful, with the younger burst accounting for the properties dominated by OB stars (neon ratio, $L_{\mathrm{Lyc}}, L_{\mathrm{bol}}$ ) and the older burst for those dominated by red supergiants $\left(L_{K}\right.$ and $\left.W_{2.29}\right)$. For regions a few tens of parsecs in size, very short burst timescales can be explained by stellar winds and supernova explosions rapidly disrupting the surrounding interstellar medium (ISM) after the onset of star formation. The spatial superposition of two distinct bursts towards individual regions can be attributed to projection effects. The red supergiants are distributed in the disk and concentrated around the nucleus while the $\mathrm{HII}$ regions lie mainly along a circumnuclear ring; given the nearly edge-on orientation of M 82, the corresponding burst populations contribute to the properties measured along each line of sight within the 3D field of view.

Assuming two successive short bursts $\left(t_{\mathrm{sc}}=1 \mathrm{Myr}\right)$, we modeled all individual resolution elements in our $3 \mathrm{D}$ data to constrain the burst age and intensity. The resulting star formation history, obtained by integrating the initial star formation rate $R_{0}$ per age bin, is plotted in Figure 3. Two distinct starburst episodes are outlined, each of duration of a few $10^{6} \mathrm{yr}$, peaking 4.5 and $9 \mathrm{Myr}$ ago. The spatial distributions of burst ages and intensities indicate a roughly 
constant age for each burst population, with the older burst having been particularly intense within $50 \mathrm{pc}$ of the nucleus while the younger burst was concentrated at larger radii coinciding with the ring of ionized gas. The low $M / L_{K}$ ratio and the presence of young supernova remnants further support lower level activity out to radii of $\sim 300 \mathrm{pc}$ during the older episode. Our spatially detailed modeling suggests that each starburst episode in the central $500 \mathrm{pc}$ of M 82 was triggered nearly simultaneously, presumably due to large gas inflows and brief compression events, and decayed substantially within a few $10^{6} \mathrm{yr}$ locally due to strong negative feedback effects, resulting in a similarly rapid decline of the global star formation activity.

\section{Earlier history of M 82: the fossil starburst regions}

Recently, de Grijs et al. (2001) investigated the properties of the compact luminous stellar clusters and individual bright giant stars resolved in optical and near-infrared HST imaging of a disk region $\sim 1 \mathrm{kpc}$ northeast of the nucleus. The presence of an intermediate age population at this location has previously been inferred from optical spectroscopy (e.g., Marcum \& O'Connell 1996), showing high-order Balmer absorption lines and a prominent Balmer break. From evolutionary synthesis modeling, de Grijs et al. (2001) found cluster ages ranging from $\sim 30 \mathrm{Myr}$ to over $10 \mathrm{Gyr}$ and derived ages for the brightest giants of 20$30 \mathrm{Myr}$. The cluster age distribution reveals a peak around $650 \mathrm{Myr}$ with full width of $\sim 500 \mathrm{Myr}$ and very few clusters younger than $300 \mathrm{Myr}$, delineating an earlier "extranuclear" starburst episode of comparable amplitude as the recent ones in the starburst core.

\section{Lessons from the starburst Rosetta Stone}

Simple considerations of timescales allow us to link the starburst history of M 82 with possible mechanisms driving its evolution (as discussed in detail by de Grijs et al. 2001 and Förster Schreiber et al. 2002). Numerical simulations of the M 81/M 82/NGC 3077 group date the last close passage between M 82 and M 81 at $200 \mathrm{Myr}$ ago (e.g., Yun, Ho \& Lo 1994; Yun 1999), close to the epoch of enhanced cluster formation in the fossil starburst regions. The orbital period at radii of 1000,500 , and $85 \mathrm{pc}$ is of $\sim 100,20$, and $5 \mathrm{Myr}$, corresponding fairly well to the 'outside-inside-out' propagation of the starburst episodes. Comparison of the cumulative mechanical energy injected into the ISM (derived from our models) and its gravitational binding energy suggests the collective effect of stellar winds and supernova explosions is able to disrupt the current gas reservoir in the central $500 \mathrm{pc}$ on timescales of $\$ 10^{6} \mathrm{yr}$, supporting feedback effects as plausible efficient quenching mechanism for the two recent bursts.

The following scenario can be proposed for M 82: (i) As a result of the interaction with $\mathrm{M} 81 \sim 10^{8} \mathrm{yr}$ ago, the ISM in M 82 experienced strong large-scale torques, loss of angular momentum, and important infall towards the nuclear regions, leading to enhanced star formation activity in the central kiloparsec. The stellar bar, likely induced by the interaction, may have played a role in channeling the inflow; depending on the details of the bar development, the infalling ISM can reach the nucleus or be trapped in circumnuclear regions due 
to dynamical resonances; (ii) A first starburst episode took place $650 \mathrm{Myr}$ ago at radii of $\sim 1 \mathrm{kpc}$. Enhanced star formation in these regions subsided about $300 \mathrm{Myr}$ ago and was suppressed in the last 20 - 30 Myr. Bar-related resonances and self-gravity may have prevented substantial dispersion of the burst site over the past several rotation periods; (iii) A starburst episode followed 8-15 Myr ago throughout the inner $500 \mathrm{pc}$ and was rapidly exhausted. The central few tens of parsecs at the nucleus hosted the most intense star formation activity; and (iv) A subsequent episode 4-6 Myr ago was triggered predominantly by bar-induced resonances, taking place mainly in a circumnuclear ring of radius $\sim 85 \mathrm{pc}$ and further out along the bar, and also decayed rapidly.

A more complete picture of starburst activity in M82 has emerged from the combination of results for both the active starburst core and the outer poststarburst disk regions, revealing its episodic behaviour and complex spatial progression. The case of M 82 emphasizes the importance of both detailed and global approaches for constraining the overall starburst history in galaxies, and the interplay between large-scale dynamical mechanisms and feedback processes determining the evolution of starburst activity.

Acknowledgments. We gratefully acknowledge the organizers of this symposium and the IAU for financial support.

\section{References}

de Graauw, Th., Haser, L.N., Beintema, D.A., et al. 1996, A\&A (Letters) 315, L49

de Grijs, R., O'Connell, R.W., Gallagher, J.S. 2001, AJ 121, 768

Ferland, G.J. 1996, HAZY, a Brief Introduction to CLOUDY (Department of Physics \& Astronomy, University of Kentucky: Internal Report)

Förster Schreiber, N.M. 2000, AJ 120, 2089

Förster Schreiber, N.M., Genzel, R., Lutz, D., Kunze, D., Sternberg, A. 2001, ApJ 552, 544

Förster Schreiber, N.M., Genzel, R., Lutz, D., Sternberg, A. 2002, ApJ submitted

Freedman, W.L., Madore, B.F. 1988, ApJ (Letters) 332, L63

Kessler, M.F., Steinz, J. A., Anderegg, M.E., Clavel, J., Drechsel, G., Estaria, P., Faelker, J., Riedinger, J.R., Robson, A., Taylor, B.G., Ximenez de Ferran, S. 1996, A\&A (Letters) 315, L27

Marcum, P., O'Connell, R.W. 1996, in: C. Leitherer, U. Fritze-von Alvensleben, J. Huchra (eds.), From Stars to Galaxies: The Impact of Stellar Physics on Galaxy Evolution, ASP-CS 98, 419

Origlia, L., Moorwood, A.F.M., Oliva, E. 1993, A\&A 280, 536

Pauldrach, A.W.A., Hoffmann, T.L., Lennon, M. 2001, A\&A 375, 161

Rieke, G.H., Lebofsky, M.J., Thompson, R.I., Low, F.J., Tokunaga, A.T. 1980, ApJ 238, 24

Rieke, G.H., Loken, K., Rieke, M.J., Tamblyn, P. 1993, ApJ 412, 99

Satyapal, S., Watson, D.M., Pipher, J.L., Forrest, W.J., Greenhouse, M.A., Smith, H.A., Fisher, J., Woodward, C.E. 1997, ApJ 483, 148

Schaller, G., Schaerer, D., Meynet, G., Maeder, A. 1992, A\&AS 96, 269

Sternberg, A. 1998, ApJ 506, 721

Telesco, C.M. 1988, Ann. Review Astron. Astrophys. 26, 343

Telesco, C.M., Gezari, D.Y. 1992, ApJ 395, 461 
Weitzel, L., Krabbe, A., Kroker, H., Thatte, N., Tacconi-Garman, L.E., Cameron, M., Genzel, R. 1996, A\&AS 119, 531

Yun, M.S. 1999, in: J.E. Barnes \& D.B. Sanders (eds.), Galaxy Interactions at Low and High Redshift, Proc. IAU Symp. No. 186 (San Francisco: ASP), 81

Yun, M.S., Ho, P.T.P., Lo, K.Y. 1994, Nature 372, 530

\section{Discussion}

LEITHERER: Can we hope to obtain near-IR spectra at $R \approx 10000$ to resolve the CO features in the $H$ - and $K$-bands? The controversy on the low-mass end of the IMF is still unsettled and a virial mass determination could answer this question.

SCHREIBER: The issue of low-mass IMF in starbursts is indeed very crucial. In M82, I am aware of one group working on this topic and trying to use NIRSPEC at the Keck Telescope to determine dynamical masses of clusters in the nuclear regions, but results have not been announced yet. I think it is difficult because of sensitivity limitations. Smith \& Gallagher (1999, AAS 194, 1203) have studied cluster ' $F$ ' based on optical data, and conclude there might be indications for an IMF biased against the formation of low-mass stars, but this remains to be confirmed with improved data.

Johnson: Two comments. (i) Based on the radio data of Kronberg et al. (2000, ApJ 535, 706), there don't appear to be any compact thermal radio sources (we may not be able to disentangle them from all the non-thermal emission). If there really aren't any, this implies that star-formation is currently shut off and perhaps we are seeing some kind of regulation mechanism which is very interesting. (ii) This comment is really for all of the speakers this afternoon. I think we need to be very careful about what we call a 'super' star cluster, so that we aren't calling objects 'super' which are really just very impressive.

SCHREIBER: $(i)$ These results are consistent with our findings from the modeling, that support very short timescales for the most recent starburst episodes in the central $500 \mathrm{pc}$ of M 82 of about a few million years only. The last burst peaked at about $5 \mathrm{Myr}$ ago and has declined very rapidly in intensity, with little of any current star formation still on-going. ( $i i)$ Masses inferred for the clusters resolved with HST-WFPC2 in M 82 range from $\sim 10^{4}$ to $\sim 10^{6} \mathrm{M}_{\odot}$. The $\sim 10^{6} \mathrm{M}_{\odot}$ clusters can be called 'super', but I suppose at the lower range, it becomes less clear and a matter of personal choice in definition. I agree that a consensus in definition should be established.

STEVENS: What are the relative (absolute) sizes of the two recent burst in M 82 ?

SCHREIBER: The two most recent bursts in the starburst core of M 82 (central $500 \mathrm{pc}$ ) were of comparable intensity in terms of mass of stars formed. Assuming two exponentially decaying bursts with a timescale of $1 \mathrm{Myr}$, the older burst $(\sim 10 \mathrm{Myr})$ produced a factor of 2-4 more mass than the younger burst ( $\sim 5 \mathrm{Myr})$. Absolute masses are dependent on the assumed lower mass cutoff of the IMF. For a Salpeter IMF from $1 \mathrm{M}_{\odot}$ to $100 \mathrm{M}_{\odot}$, we find about $4 \times 10^{7} \mathrm{M}_{\odot}$ and $1.6 \times 10^{8} \mathrm{M}_{\odot}$ for the youngest and oldest bursts, respectively, within the starburst core. 